\title{
A Practical Framework for 802.11 MIMO Rate Adaptation
}

\author{
Lara Deek* ${ }^{*}$ Eduard Garcia-Villegas ${ }^{\ddagger}$, Elizabeth Belding ${ }^{\dagger}$, Sung-Ju Lee ${ }^{\S}$, Kevin \\ Almeroth $^{\dagger}$ \\ University of Illinois at Urbana-Champaign* , UPC-BarcelonaTECH ${ }^{\ddagger}$, UC Santa Barbara $^{\dagger}$, KAIST $^{\S}$ \\ laradeek@illinois.edu, eduardg@entel.upc.edu, ebelding@cs.ucsb.edu, \\ sjlee@cs.kaist.ac.kr, almeroth@cs.ucsb.edu
}

\begin{abstract}
The emergence of MIMO antennas and channel bonding in $802.11 \mathrm{n}$ wireless networks has resulted in a huge leap in capacity compared with legacy 802.11 systems. This leap, however, adds complexity to optimizing transmission. Not only does the appropriate data rate need to be selected, but also the MIMO transmission technique (e.g., Spatial Diversity or Spatial Multiplexing), the number of streams, and the channel width. Incorporating these features into a rate adaptation (RA) solution requires a new set of rules to accurately evaluate channel conditions and select the appropriate transmission setting with minimal overhead. To address these challenges, our contributions in this work are two-fold. First, we propose a practical link metric that accurately captures channel conditions in MIMO 802.11n environments, and we call this metric diffSNR. Using diffSNR captured from real testbed environments, we build performance models that accuractely predict link quality in $95.5 \%$ of test cases. Practicality and deployability are guaranteed with diffSNR as it can be measured on all off-the-shelf MIMO WiFi chipsets. Second, we propose ARAMIS (Agile Rate Adaptation for MIMO Systems), a standard-compliant, closed-loop RA solution that jointly adapts rate and bandwidth, and we utilize the diffSNR-based 802.11n performance models within ARAMIS's framework. ARAMIS adapts transmission rates on a perpacket basis; we believe it is the first closed-loop, 802.11 RA algorithm that simultaneously adapts rate and channel width. We have implemented ARAMIS with diffSNR on Atheros-based devices and deployed it on our 15-node testbed. Our experiments show
\end{abstract}

Preprint submitted to Elsevier

February 9, 2015

(C) 2015. This manuscript version is made available under the Elsevier user license http://www.elsevier.com/open-access/userlicense/1.0/ 
that ARAMIS accurately adapts to a wide variety of channel conditions with negligible overhead. Furthermore, ARAMIS outperforms existing RA algorithms in 802.11n environments with up to a 10 fold increase in throughput.

Keywords: IEEE 802.11n, Rate Adaptation, Channel Bonding, Measurement,

Performance, Experimentation.

\section{Introduction}

Rate adaptation (RA) selects the best physical bitrate based on time-varying channel qualities. With the emergence of the IEEE 802.11n standard, WiFi technologies have witnessed a significant increase in sophistication and complexity that require novel approaches to RA. RA in 802.11 networks not only needs to choose the operating rate, but also the channel width and MIMO mode. Using MIMO, a solution can send a single stream using spatial diversity to improve signal strength, or multiple simultaneous streams using spatial multiplexing to increase the transmission rate.

Identifying a link metric that accurately characterizes and exploits 802.11n MIMO link performance is an important component of an effective RA solution. Perhaps the best RA solution for MIMO environments is to use 802.11n's Channel State Information (CSI) feedback from the receiver to compute the transmission rate. However, complete CSI information is costly to obtain and store [1] and is therefore supported by very few 802.11 n devices. Existing RA solutions adopt a practical approach and use a credit-based system [2] or rate sampling [3, 4, 5]. Instead of adapting the rate based on understanding the impact of environment conditions on $802.11 \mathrm{n}$ features, these solutions rely on certain heuristics to converge to the best rate, which can be costly or misdirected. Therefore, there is a clear need to build RA solutions over a new, practical link metric that accurately characterizes links in MIMO environments.

20 To characterize MIMO link performance and capture channel conditions, particularly for the majority of systems where CSI is not available, our previous work developed a practical link metric called diffSNR, which provides a good balance between implementability and accuracy [6]. Similar to CSI-based metrics [7], diffSNR also provides the flexibility to predict performance for a given rate and channel width com- 
bination simultaneously. diffSNR is computed as the difference between the best and the worst SNR (Signal-to-Noise Ratio) at any of the receiver's antennas; it reveals insights on the nature of signal reception, i.e. whether signals combine constructively or destructively at the receiver's antennas. Our close analysis revealed the dependency of performance on diffSNR, and we exploit this relationship in the design of a measurement-driven link quality predictor. Through testing in a variety of environments, we showed that our diffSNR-based link predictor estimates link quality over all supported rate and bandwidth combinations with an accuracy of at least $95.5 \%$.

A natural extension is to evaluate the application and impact of diffSNR on RA by implementing or incorporating diffSNR in the context of an effective $802.11 \mathrm{n}$ RA framework. There are two main approaches to RA one can adopt: an open-loop and a closed-loop approach. In open-loop RA, the transmitter estimates the best rate of the link to the receiver by building on some set of parameters or metrics measured at the transmitter [8]. A closed-loop RA is one in which the receiver's insight into the channel conditions contributes to determining the rate.

As networks become more complex, the use of open-loop RA techniques becomes increasingly inaccurate. An RA solution now has to account for many variables that a transmitter alone cannot accurately capture. In legacy clients, RA mechanisms have to choose among four PHY rates in 802.11b and eight rates in 802.11a/g, whereas $802.11 \mathrm{n}$ allows at least 64 combinations (32 rates x 2 channel widths) and 802.11ac multiplies 45 this number by four. By allowing the receiver to contribute to the RA process, we gain an accurate understanding of environment conditions, and the transmitter can more efficiently select the appropriate rate for the link [7].

In a closed-loop RA model, the receiver's insight into channel conditions is used to compute the transmission rate. A feedback mechanism should therefore be incorporated into the design. In fact, the 802.11n standard supports an explicit feedback system in MCS Request and MCS Feedback [9]. By exploiting this standard-compliant feedback mechanism, accurate receiver-based RA solutions can be designed for $802.11 \mathrm{n}$ MIMO environments.

The state of the art for RA in 802.11n calls for a standard-compliant, closedloop solution that accurately exploits the new features in 802.11 MIMO environments. 
Therefore, the RA solution must adopt a link metric that accurately characterizes MIMO link performance. We propose such an RA solution, which we call ARAMIS (Agile Rate Adaptation for MIMO Systems) [6].

ARAMIS is a closed-loop, per-packet RA solution that simultaneously adapts both rate and channel width. In our previous work on channel bonding, we showed the importance of adapting bandwidth in 802.11n with RA to maximize performance [10, 11]. ARAMIS incorporates a measurement-based, $802.11 \mathrm{n}$ link predictor in its design. Given the current channel conditions, the link predictor estimates Packet Reception Rate (PRR) for the given link at all supported rate and bandwidth combinations. Using this information, ARAMIS then selects the best operating point, and sends the feedback to the transmitter using a standard-compliant mechanism. We use our proposed metric diffSNR to characterize MIMO link performance and capture channel conditions, as well as serve as input to the link predictor.

We implemented ARAMIS with diffSNR and evaluated it on a 15-node testbed [6]. We compared ARAMIS to leading RA solutions for 802.11n, namely Ath9k [5], Minstrel HT [4], and RAMAS [2]. We evaluated the solutions under various scenarios, including interference, mobility, and hidden nodes. We demonstrated that ARAMIS is robust, consistently performs well and outperforms existing solutions, with an average of 0.5 fold and up to a 2.87 fold increase in throughput compared to its best competitor, 75 RAMAS, and an average of 3.85 and up to a 10 fold increase compared to Ath9k.

We further provide a more detailed evaluation of ARAMIS in two respects. First, we compare ARAMIS against an ideal solution. This comparison allows us to gauge how closely ARAMIS approximates a performance upper bound. To conduct this evaluation, we use a trace-driven simulation, where we implement both ARAMIS with diffSNR and an ideal solution. We find that ARAMIS closely approximates the ideal by taking advantage of per-packet processing. Per-packet RA enables quick and fine adjustments to varying channel conditions and allows the exploitation of narrow windows of higher bandwidth opportunities. Ideally, we would compare ARAMIS to an existing solution in literature that uses CSI to perform rate adaptation [7]. However, we are unable to implement CSI-based RA solutions, since our chipsets do not support CSI. We believe, however, that the ideal solution evaluated in the trace-driven simulations 
provides a more accurate upper bound, since it is implementation-independent.

Our second contribution to a detailed evaluation is of ARAMIS's link predictor that is built over diffSNR. This evaluation reveals that the link predictor exhibits relatively consistent behavior for different frame sizes and rates. Not surprisingly, the performance of aggressive modulations is more difficult to predict, and this is translated into higher Packet Reception Rate (PRR) prediction errors. Furthermore, PRR prediction errors increase when spatial multiplexing is used. Notwithstanding, ARAMIS's link predictor maintains a reasonable level of accuracy, with an average absolute error in PRR predictions of $12 \%$, and by adding a training mechanism, errors fall to $5.8 \%$.

A final contribution of our work is a detailed evaluation of the performance insights SNR provides in 802.11n MIMO environments. Though the inaccuracies of SNR have been identified in prior work [7, 12], we show that SNR measurements are still useful to provide a high-level assessment of the channel. Through our detailed evaluation of SNR behavior, we come to understand the impact of different 802.11n features, namely spatial multiplexing, spatial diversity, and channel bonding, on its performance.

This paper is organized as follows. We first evaluate the efficacy of SNR and our metric diffSNR in 802.11n MIMO environments in Section 2, where we also detail the implementation cost of CSI. We then present the application of diffSNR in the design of a link predictor in Section 3, and evaluate its prediction accuracy. Section 4 discusses our adopted ARAMIS rate selection algorithm and its components. We then evaluate ARAMIS with diffSNR and compare it to existing solutions under both a simulation and testbed environment in Section 5. Related areas of research are discussed in Section 6 . Finally, we conclude in Section 7

\section{Metrics for MIMO links}

We are first motivated by the need for a new metric by identifying the limitations of a commonly used and accessible link metric, RSSI (Received Signal Strength Indicator in $\mathrm{dBm}$ ), and the cost of using full CSI (Channel State Information), when available. We then present diffSNR and examine how it can be used together with RSSI to ac- 
or performance in terms of Packet Error Rate (PER, where: PER $=1-$ PRR). We conduct all experiments for both $20 \mathrm{MHz}$ and $40 \mathrm{MHz}$ channels, and we discuss our observations for three MCS indices that cover robust (MCS 8), intermediate (MCS 12), and aggressive (MCS 15) PHY rates. For each MCS, we send 5,000 1kB UDP datagrams over 50 different links, selected to cover a wide variety of cases ${ }^{1}$. For legibility, we present a subset of our results that best represents the patterns in the behavior of RSSI and diffSNR.

\subsection{The Limitation of RSSI}

RSSI, used to directly compute the Signal-to-Noise Ratio (SNR) in $\mathrm{dB}^{2}$, has traditionally been used to represent the quality of a link [13]. With knowledge of SNR, and assuming a channel with AWGN noise (additive white Gaussian noise), empirical curves or known theoretical formulas have been used to infer the bit error ratio (BER) for any given MCS. With the BER and the transmitted frame length, an upper bound for the packet error rate (PER) can then be estimated. The existing models that map RSSI to performance show that a link's PER is 1.0 for sufficiently low RSSI and then steeply drops to 0.0 as RSSI increases beyond a threshold value [14].

Fig. 1 plots PER vs SNR averaged over the 50 links in our testbed. Fig. 1)(a) plots the values for one transmit stream and Fig. 1.b) for two streams. As RSSI increases, we expect PER to drop since the receiver can better decode the received signal. Fig. 1, however, shows that this is not necessarily the case. When we compare Figs. 11(a) and 1.b), we observe irregular behavior particularly for aggressive modulation schemes with spatial multiplexing (MCS 12 and 15). PER does not converge to 0 for high SNR and surprisingly in Fig. 1.b), performance degrades for SNR > 55dB; that is, contrary to what seems to be an established dogma among many network administrators, higher transmitter power does not translate to better reception.

There are two explanations to this behavior. High SNR values are achieved when

\footnotetext{
${ }^{1}$ See Section 5 for testbed details.

${ }^{2} 802.11$ A Atheros driver assumes a predefined noise floor and thus computes RSSI $=$ SNR + NoiseFloor, where SNR is measured from the channel, and NoiseFloor is set to a default, predefined value of $-95 \mathrm{dBm}$. We therefore use RSSI and SNR interchangeably, as the latter is a scaled version of the former.
} 
the output power is high and/or when the propagation losses are low due to the close proximity of the transmitter/receiver pair in the absence of obstacles. Therefore, one explanation is that high output power can be the source of constellation errors when using OFDM. The combination of OFDM and high order amplitude modulations (such as 64-QAM used in MCS 5 to 7 and 13 to 15) is prone to high peak-to-average ratios: high peaks cause the power amplifiers to move toward saturation [15], exhibiting nonlinear behavior that produces inter-modulation distortion. However, the anomaly is observed for MCS 12 and 15, but not for MCS 7.

Therefore, the answer must be the presence of a dominant path between a transmitter/receiver pair, such as when the nodes are close to each other in direct line-ofsight, which results in a high Rician $K$-factor and the channel becomes increasingly correlated in space. This hampers the utilization of spatial multiplexing [16], since it requires that simultaneous streams follow independent paths with sufficiently different spatial signatures.

Fig. 1 also shows that SNR is a poor indicator of link quality for different channel widths. For the same SNR, a $40 \mathrm{MHz}$ channel suffers a higher PER. Wider transmissions are more likely to suffer from frequency selective fading, which causes SNR variations across the OFDM subcarriers, and PER is dominated by the lower SNR carriers. A $40 \mathrm{MHz}$ channel, therefore, not only requires a stronger transmission power to achieve the same SNR [17] but also a higher SNR to provide the same PER.

Despite those anomalies, Fig. 1 seems to show clear transitions between usable and unusable links for low MCS indices. However, if we observe the raw data used to obtain the average behavior depicted in Fig. 1, those transitions are less apparent. Figs. 2 and 3 plot per-link PER vs SNR for all testbed links and bandwidths. On the Y-axis, each point represents PER measured over 5,000 frame transmissions in one particular link. On the X-axis, each point is the average SNR of the received stream of packets. Fig. 2 shows results for a single stream, while Fig. 3 for two streams.

Figs. 2 and 3 show that RSSI is a reliable metric when robust MCS modes are used that exploit spatial diversity. For example, the transition region for MCS 0 is only 3$4 \mathrm{~dB}$ wide; for a given link at MCS 0 , if the measured SNR is below $6 \mathrm{~dB}$, the link is infeasible $(\mathrm{PER} \approx 1)$ and, if the SNR is above $10 \mathrm{~dB}$, it is feasible (PER $\approx 0$ ). How- 


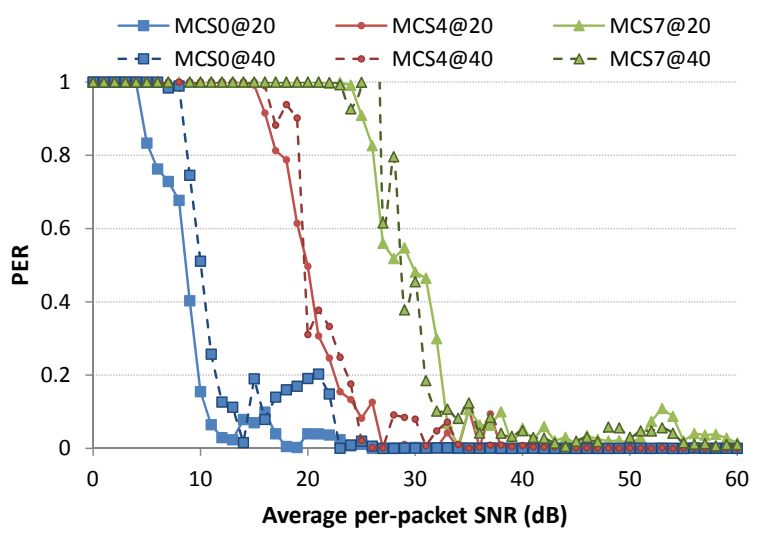

(a) One transmit stream.

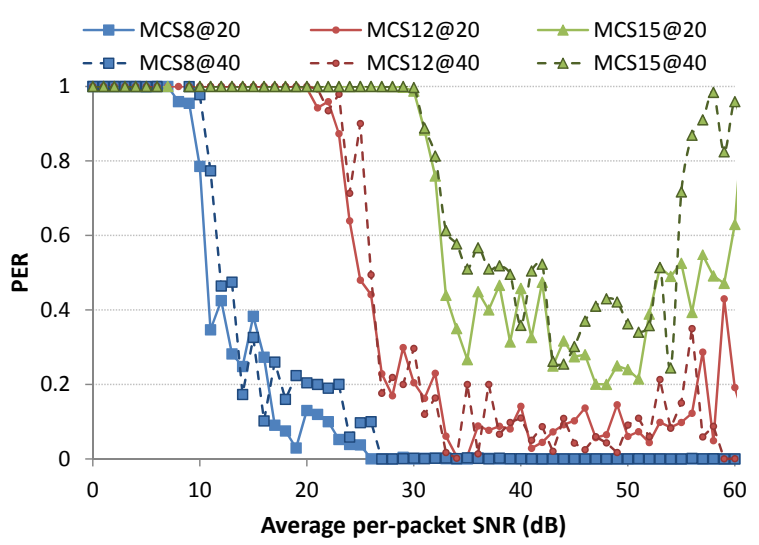

(b) Two transmit streams.

Figure 1: Average PER and per-packet SNR over testbed links.

ever, for SNR values between 5 and $10 \mathrm{~dB}$, the feasibility of a link is uncertain; some links yield an excellent performance with an SNR of 6dB, while others are not feasible with a higher SNR of $10 \mathrm{~dB}$. This uncertainty is amplified with spatial multiplexing and more aggressive modulations, where the transition region between a feasible and an infeasible link becomes wider. For example, when both spatial multiplexing and moderate or fast PHY rates are used (e.g. MCS $\geq 12$ ), the transition region could be as wide as $35 \mathrm{~dB}$ ! In such cases, RSSI alone does not provide sufficient information to assess the feasibility of a link. This result is consistent with previous work [12, 7]. 


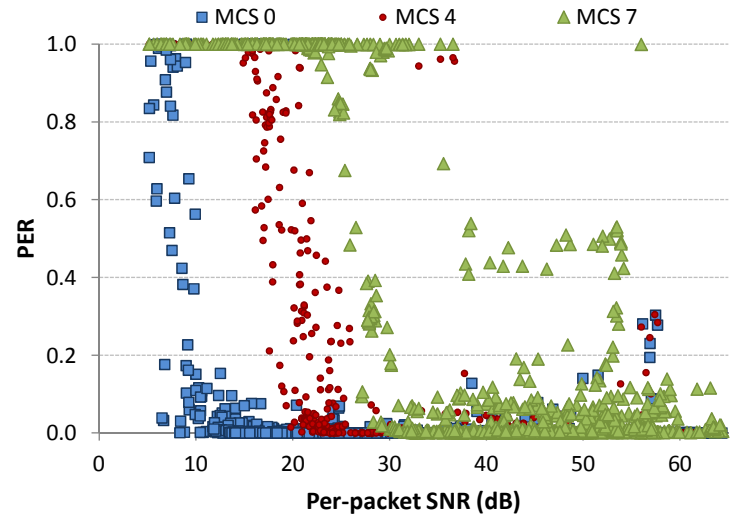

(a) $20 \mathrm{MHz}$ wide channel.

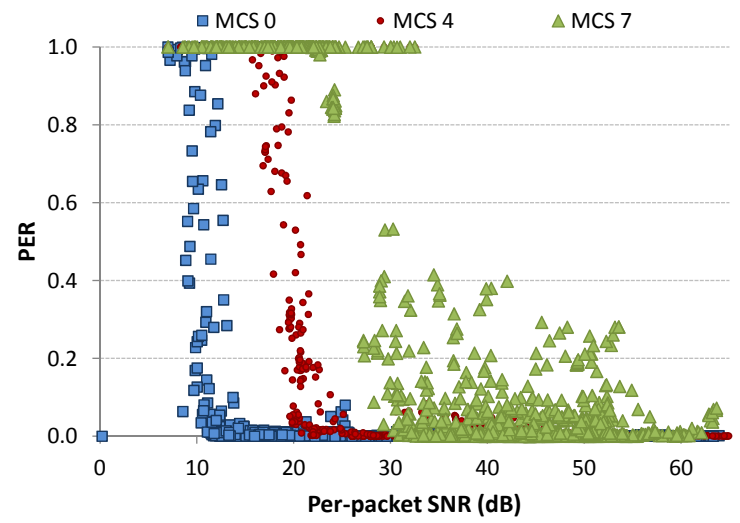

(b) $40 \mathrm{MHz}$ wide channel.

Figure 2: Per-link PER vs SNR measurements for one stream.

\subsection{Channel State Information (CSI)}

CSI describes the current channel conditions with fine granularity ${ }^{3}$ It consists of the attenuation and phase shift for each spatial stream to every receive antenna, for every OFDM subcarrier (56 subcarriers for a $20 \mathrm{MHz}$ bandwidth and 114 for a $40 \mathrm{MHz}$ bandwidth in $802.11 \mathrm{n}$ ). Measuring a complete and timely CSI for all possible MIMO channel configurations requires excessive sampling overhead [1].

\footnotetext{
${ }^{3}$ Here, we refer to the full CSI matrix and not the coarse grained CSI provided by Intel $802.11 \mathrm{n}$ chipsets [7].
} 


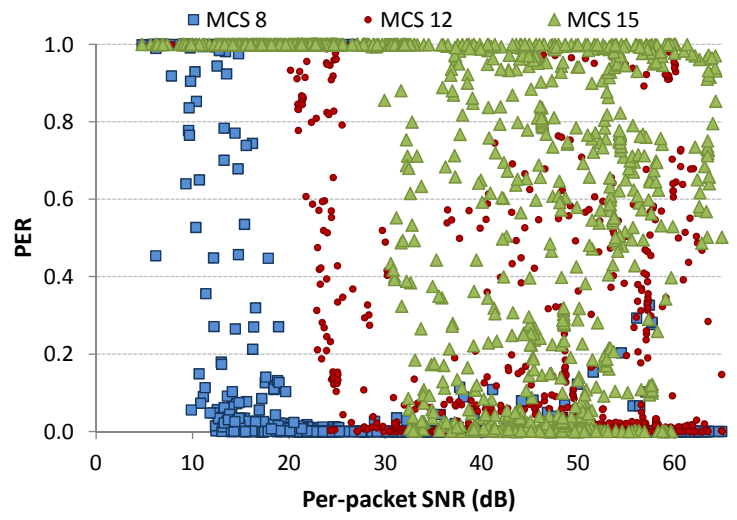

(a) $20 \mathrm{MHz}$ wide channel.

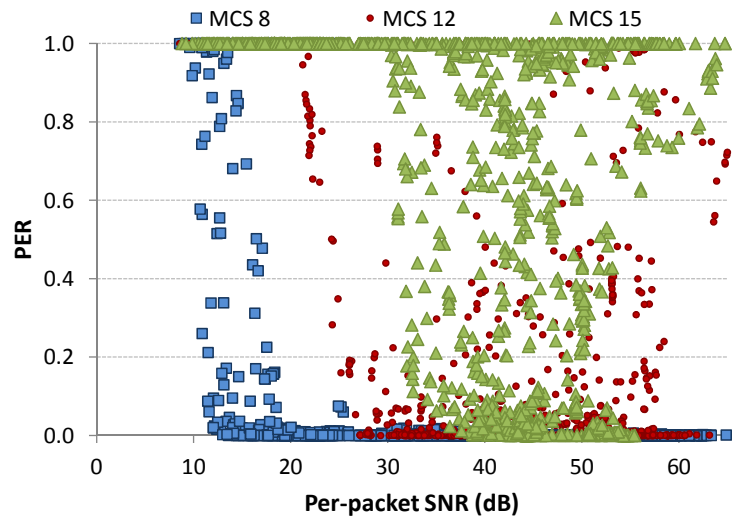

(b) $40 \mathrm{MHz}$ wide channel.

Figure 3: Per-link PER vs SNR measurements for two streams.

In some implementations, successful decoding of a data packet is required to compute CSI [7]. Additionally, for a $T \times R$ MIMO system of bandwidth $W$, a series of probe frames must be sent using $T$ transmit antennas over a bandwidth $W$, and received over $R$ receive antennas to obtain the complete $T \times R \times W$ CSI matrix. For example, a $3 \times 3$ MIMO system allows transmissions using one, two or three simultaneous data streams 4 , and thus the complete CSI requires probing all combinations of number of streams and transmit antennas. As a result, current CSI estimation approaches require

${ }^{4}$ Where the maximum number of supported data streams in a $T \times R$ MIMO system is $\min (T, R)$. 
seven probes (or samples) to obtain CSI for all possible configurations of a $3 \times 3 \mathrm{MIMO}$ system. Single-stream MCSs require three probes, to collect CSI for each individual transmit antenna. Similarly, two-stream MCSs require three probes, to collect CSI for each combination of two transmit antennas. Finally, three-stream MCSs require a single probe from a transmission from all three transmit antennas. The number of required probes increases dramatically with more antennas and wider channel widths.

Communicating the computed CSI matrix in a feedback packet also consumes significant bandwidth overhead. The size in bytes of a feedback packet with complete, noncompressed CSI is $1.029 \mathrm{~KB}$ for a $20 \mathrm{MHz}$ channel and $2.095 \mathrm{~KB}$ for a $40 \mathrm{MHz}$ channel [9]. Based on channel coherence time [18], CSI at the transmitter needs to be updated at least once every 50ms. CSI feedback, as a result, consumes $160.64 \mathrm{~Kb} / \mathrm{s}$ to $335.2 \mathrm{~Kb} / \mathrm{s}$ respectively. In a per-packet RA implementation, where CSI needs to be updated frequently, the bandwidth consumed by CSI feedback quickly becomes a significant overhead.

Complete CSI is clearly expensive to obtain and communicate, and therefore its applicability to a per-packet RA solution, particularly in dynamic environments where timely channel information is necessary, is limited. Our aim is, therefore, to identify an alternative MIMO link metric in the design of an agile rate adaptation mechanism.

\subsection{Differential SNR (diffSNR)}

It is clear that RSSI alone does not accurately capture the factors that cause the variability in 802.11 channels. Frequency selectivity due to multipath is one major factor whose effects are only captured using OFDM per-subcarrier SNR information [7]. Antenna correlation, or spatial selectivity, is another factor [19]. Both factors, however, require costly CSI which is supported by only very few devices [1]. For devices that do not support CSI, we develop a practical metric, called diffSNR, by using the channel metrics available to us in all commodity MIMO devices. We now show how we can use diffSNR to accurately reflect channel quality in $802.11 \mathrm{n}$ networks.

Multipath propagation in wireless environments produces constructive and destructive interference at the receiving antennas [20]. The resulting signal combination varies at different locations, a concept referred to as spatial selectivity. MIMO systems take 
advantage of these multipath phenomena to improve performance.

When received signals combine destructively in a process called selective fading, SNR can degrade and will reliably indicate a lossy link. Since per-packet SNR is the linear sum of all per-antenna measurements, if only a portion of the antennas experience fading, the reported SNR may be high even though the link could be lossy. Reported SNR does not reflect the extent of selective fading. We therefore argue that knowledge of the SNR combined with the per-antenna SNR provides us with some added insight, which can be used to predict the link performance with greater accuracy. We henceforth define the difference between the best and the worst SNR at any of the receiver's antennas as diffSNR.

After analyzing real-time traces of RSSI and diffSNR in different scenarios, we observe that diffSNR does not depend significantly on either (i) the transmitter's output power, where diffSNR varies less than 6\%, (ii) the MCS used, where diffSNR varies less than $2 \%$ as shown in Fig. 4, or (iii) the channel width. On the other hand, diffSNR shows a clear dependency on the environment: factors such as rich scattering, dynamic/static positioning, line-of-sight, and obstacles. Fig. 5 5 provides two paradigmatic examples of the real-time evolution of SNR and diffSNR. We find that a static scenario exhibits fewer variations, as shown in Fig. 5(a), while a more dynamic environment is reflected in a wider dispersion of the measured diffSNR which exhibits frequent peaks, as depicted in Fig. 5(b). A peak in diffSNR can occur when RSSI increases and a subset of the antennas receive constructive interference. However, we observe that high diffSNR peaks are often ( $80 \%$ of the time) caused by some of the antennas suffering from fading; that is, there is a negative correlation between RSSI and diffSNR. This behavior is also deduced in Fig. 5. (b).

Moreover, for links with similar RSSI, we find that diffSNR can be used to characterize their performance differences. We illustrate this behavior in Fig. 6 using three representative links. We evaluate their PER vs SNR relationships using spatial multiplexing (MCS 12 and 15) and a 40MHz channel. Link 1 successfully transmits packets (PER < 0.02) using MCS 12; for MCS 15, there is a clear transition around $34 \mathrm{~dB}$ SNR. Although Link 2 has similar RSSI values to Link 1, it clearly exhibits worse performance: for MCS 12, PER increases rapidly when SNR $<30 \mathrm{~dB}$ and MCS 15 re- 


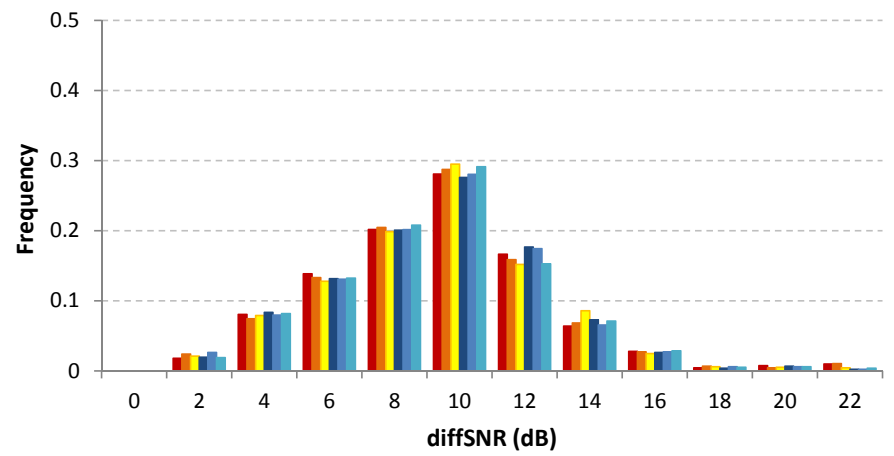

Figure 4: Normalized frequency of diffSNR measurements. Leftmost bars (red tones) represent MCS 0, 4, and 7; rightmost bars (blue tones) represent MCS 8, 12, and 15.

mains lossy until SNR $>44 \mathrm{~dB}$. The difference between Link 1 and 2 can be explained with diffSNR: for Link 1, we measure an average diffSNR of $1.82 \mathrm{~dB}$, with a standard deviation of 0.30 , while for Link 2, the average diffSNR is $9.46 \mathrm{~dB}$, with a standard deviation of 0.37. Link 3 displays the worst performance, showing an average diffSNR of $13.41 \mathrm{~dB}$. This link does not exhibit a clear transition for MCS 12 and never works for MCS 15. We can explain this behavior with the dispersion of its measured diffSNR values with a standard deviation of 0.97 .

Given the predictable behavior of diffSNR and its correlation to RSSI, in the next section, we examine the implications of the (SNR, diffSNR) relationship and how it can be used to determine link quality or performance in terms of PER.

\section{Link predictions with $\operatorname{diffSNR}$}

A link predictor accurately estimates the PRR of a link for all MCS and bandwidth combinations. We now describe the methodology we use to build such a predictor, and demonstrate how it accurately predicts PRR. In case of errors, we introduce a lowoverhead training mechanism to improve accuracy.

\subsection{A measurement-based approach}

Our analysis, summarized in Section 2.3, reveals the dependency of performance on RSSI and diffSNR together. That is, the PRR(SNR, diffSNR) relationship yields 


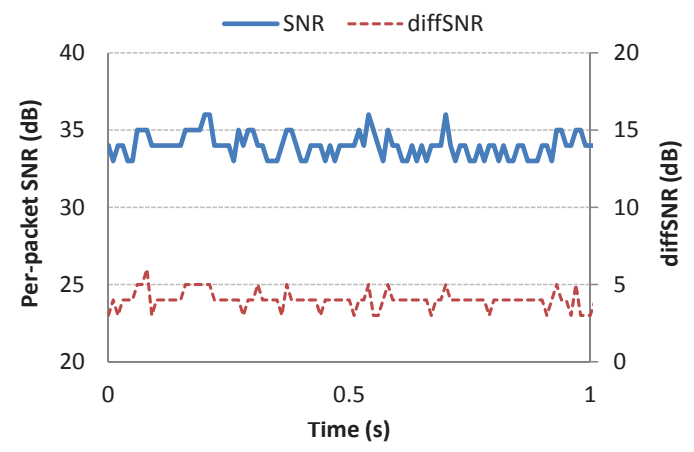

(a) Static scenario (night run) and $20 \mathrm{MHz}$ channel.

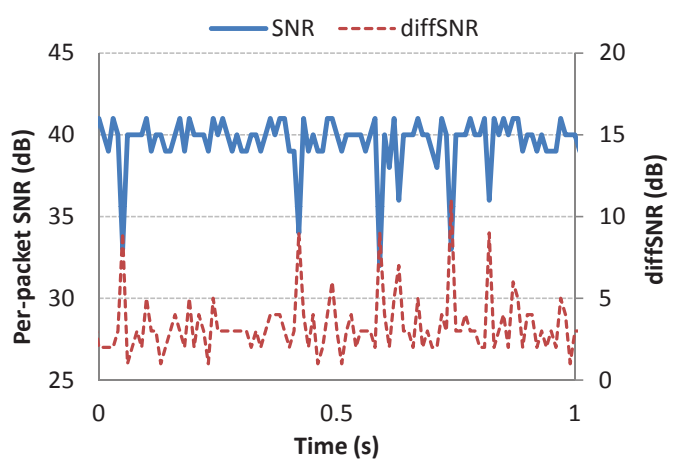

(b) Dynamic scenario (during office hours) and $20 \mathrm{MHz}$ channel.

Figure 5: Real-time evolution of per-packet SNR and diffSNR.

well-behaved surfaces that allow us to predict the PRR of a link for a given MCS and bandwidth; we show two representative graphs in Figs. 7 and 8 Fig. 7 shows the measured PRR for MCS 7 (aggressive modulation, one stream) and Fig. 8f for MCS 12 (intermediate modulation, two streams) as a function of average per-packet SNR and diffSNR. To more clearly show the transition between the links with PRR $>0.5$ and those with PRR $<0.5$, we include representative $2 \mathrm{D}$ cross-cuts of the 3D plots. For example, as shown in the projected image in Fig. 7 ( b) with an SNR of 32dB, a link with diffSNR below $5 \mathrm{~dB}$ performs well. However with a diffSNR above $10 \mathrm{~dB}$, the PRR falls to almost 0 .

Since robust modulations are less affected by fading, variations in diffSNR will be more clearly reflected on the performance of aggressive modulations. Similarly, 


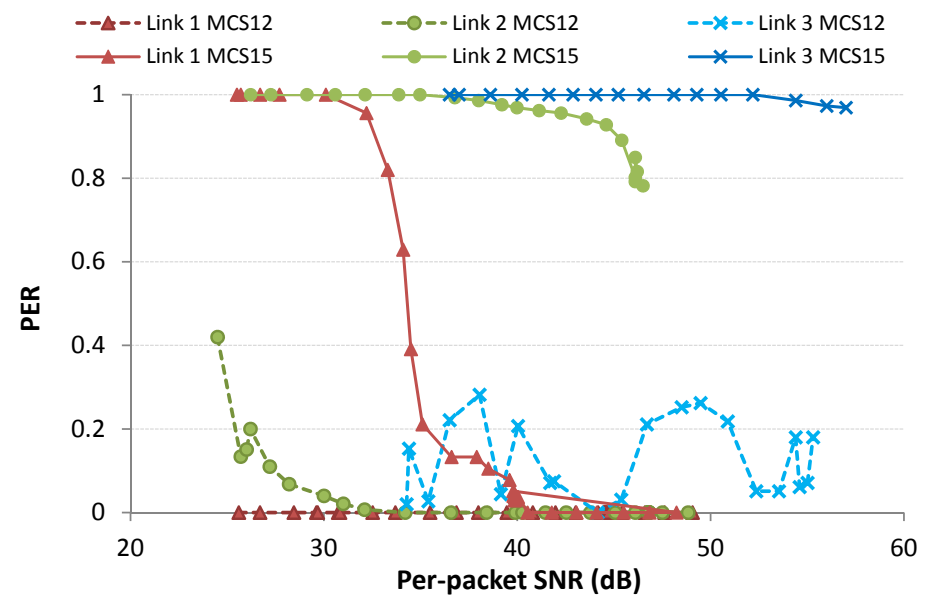

Figure 6: PER vs per-packet SNR for three links (40MHz channel).

diffSNR variations will have little impact on links with high SNR, but this impact will

increase as SNR decreases. For aggressive modulations, and particularly when multiple streams are used, predicting links with even moderate diffSNR values involves higher uncertainty. This uncertainty explains the presence of data points with PER $>0.5$ inside the "feasible" region (cf. diffSNR $>8 \mathrm{~dB}$ in Fig. 8).

From our experiments, we gather sufficient data to plot the PRR(SNR, diffSNR) surfaces for each allowable MCS. The combination of these surfaces for all MCS values constitutes our measurement-based link predictor. Our proposed predictor is thus based on a three dimensional matrix, as depicted in Fig.9. SNR and diffSNR measurements, along with the operating MCS and bandwidth of a link, constitute the matrix coordinates from which our measurement-based link predictor identifies the corresponding expected PRR for that link. It is important to note that our testbed provides us with SNR data for the control (i.e. primary) $20 \mathrm{MHz}$ channel and when channel bonding, the extended $40 \mathrm{MHz}$ channel. Predictions for $20 \mathrm{MHz}$ links can be made from measurements under 40MHz links, but not vice versa [10, 11]. Therefore, our predictor builds separate PRR surfaces for both $40 \mathrm{MHz}$ and $20 \mathrm{MHz}$ channels for each MCS.

To gather sufficient data to pre-compute and build the PRR(SNR, diffSNR) surfaces 


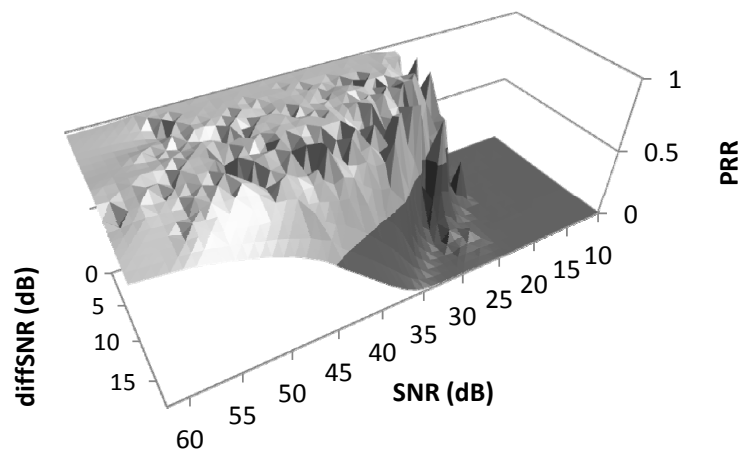

(a) $\operatorname{PRR}(\mathrm{SNR}$, diffSNR) surface.

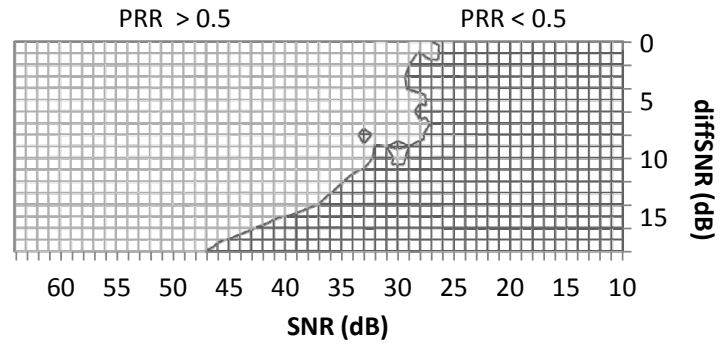

(b) $2 \mathrm{D}$ projection with isoline at $\mathrm{PRR}=0.5$.

Figure 7: PRR as a function of packet-SNR and diffSNR for MCS 7 and 20MHz channel.

for each MCS and bandwidth combination, we measure PRR(SNR, diffSNR) over all 50 testbed links while varying the transmit power from $0 \mathrm{dBm}$ to the maximum allowed power. As for the (SNR, diffSNR) data points that do not have measured values, we fill them by interpolation, using the nearest measured data points.

\section{About frame size}

Intuitively, PRR depends on the length of the frame. Hence, frame length should be accounted for to predict PRR. The PRR for any given frame size can be roughly estimated from the PRR we measure for frames carrying $1 \mathrm{kB}$ payload using the equation: $P_{x}=\left(\sqrt[L_{1000}]{P_{1000}}\right)^{L_{x}}$, where $P_{x}$ is the PRR for a payload of $x$ bytes, and $L_{x}$ is the total length (in bits) of a frame carrying an $x$ byte payload [21]. Our measurements in different scenarios consistently show that the transition regions for a link from high quality to lossy do not exhibit a noticeable difference when the payload size is changed. 


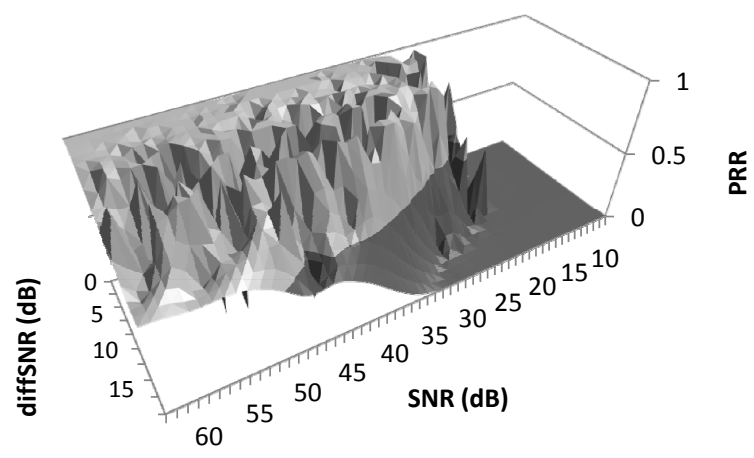

(a) $\operatorname{PRR}(\mathrm{SNR}$, diffSNR) surface.

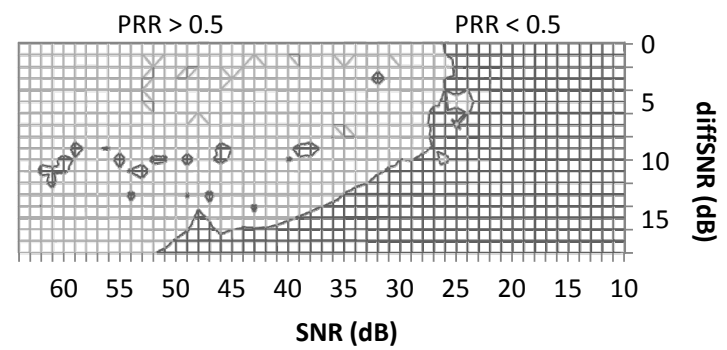

(b) $2 \mathrm{D}$ projection with isoline at $\mathrm{PRR}=0.5$.

Figure 8: PRR as a function of packet-SNR and diffSNR for MCS 12 and 20MHz channel.

Fig. 10 plots both the estimated PER, following the $P_{x}$ equation above, as well as the measured PER for payloads of 1500B (Big) and 300B (Small) for a given link using MCS 12. We observe that, as expected, larger frames show higher PER; however, the impact of frame size on the feasibility of a link is still negligible. This result indicates that transition regions do not depend on frame size, and thus we do not add frame size as an additional dimension.

\subsection{Prediction Accuracy}

The link predictor is a pre-computed matrix, with dimensions defined by the number of supported MCS, bandwidths, and the range of expected SNR and diffSNR values. To evaluate our predictor, we build two $6 \times 70 \times 20$ matrices for MCS $0,4,7,8,12$ and 15 , with SNR values from 0 to $69 \mathrm{~dB}$ and $\operatorname{diffSNR}$ values from 0 to $19 \mathrm{~dB}$, with 


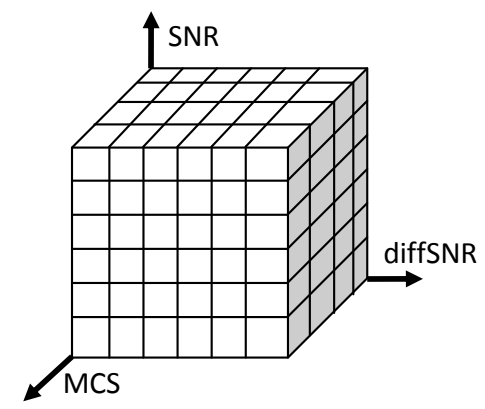

Figure 9: The 3D matrix contains measured and interpolated PER values and is the structure of our proposed link predictor.

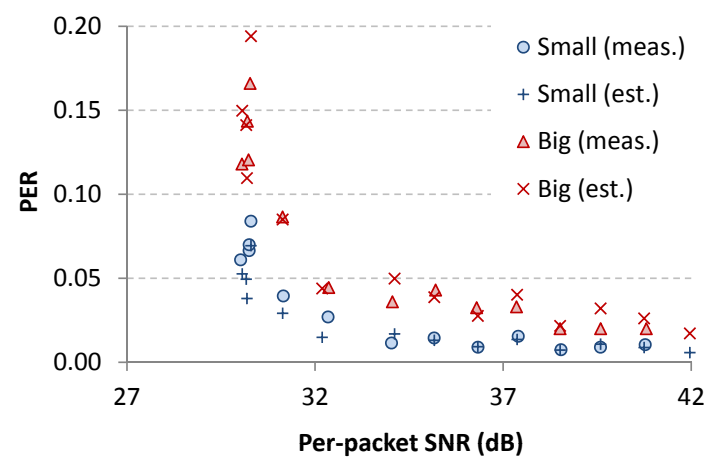

Figure 10: Measured and estimated PER vs SNR for payload size of 300B (Small) and 1500B (Big).

$1 \mathrm{~dB}$ precision 5 Our complete predictor consists of two $16 \times 70 \times 20$ matrices, and is 325 used in future sections. The reduced matrix consists of $40 \%$ of measured values (the remaining $60 \%$ are interpolated). We show that interpolation has no significant impact on the prediction accuracy.

We evaluate the accuracy of our measurement-based, pre-computed link predictor by comparing the predicted PRR values against the measured values for two different groups of transmitter/receiver pairs. The first group consists of nodes located in the same environment where the data for the predictor was collected. The second group consists of a set of laptops placed in two different off-campus small office/home office

\footnotetext{
${ }^{5}$ The distribution of diffSNR in all tested environments lie below $19 \mathrm{~dB}$.
} 
environments as well as in an outdoor environment, on a rooftop free of obstacles with direct LoS between nodes placed 20m apart. We include this second group to evaluate the utility and accuracy of the proposed predictor in unfamiliar and dissimilar environments.

For the first group of nodes located in a familiar environment, the average absolute error in PRR predictions, computed as the difference between the measured PRR and the predicted PRR, is only $4.8 \%$. This error ratio increases for high order modulations (up to $11 \%$ for MCS 15 ) since these modulations show a higher degree of uncertainty. Although the absolute error may be relatively high for some MCS indices, we reliably predict link feasibility with a $96.1 \%$ accuracy ${ }^{6}$ As for the second group of nodes in new environments, the average absolute error in PRR predictions is $12 \%$ and the accuracy in feasibility predictions is $88.1 \%$. These results show the importance of a calibration or training mechanism. To increase the prediction accuracy, we include the error of previous measurements in the new PRR predictions such that:

$$
P R R_{k}^{m, B}=\operatorname{PRR}\left(m, B, S N R_{k}, \operatorname{diffSNR} R_{k}\right)+E_{k-1}^{m, B}
$$

where $P R R_{k}^{m, B}$ is the predicted PRR for MCS $m$ and bandwidth $B ; S N R_{k}$, and diff $S N R_{k}$ are the currently measured RSSI and diffSNR values; and $P R R(w, x, y, z)$ returns a PRR value from the predictor using the input parameters. Finally, $E_{k-1}^{m, B}$ is the error in previous predictions for the same MCS and bandwidth, where $0 \leq E_{k-1}^{m, B} \leq 1$. As detailed in Section 4.2.3, we compute the error by tracking the real PRR from received data frames (i.e. no extra signaling is required) and comparing it with the predicted PRR. The value $E_{k}^{m, B}$ is computed as an exponential moving average of the measured error samples with a configurable $\alpha$. A large $\alpha$ allows the prediction mechanism to adapt faster to rapidly varying channels (e.g. with user mobility), while smaller $\alpha$ values improve the accuracy of the error estimation in more stable environments.

We re-evaluate our results in the new environments using Eq. 1. Fig. 11. a) computes the average absolute error in PRR predictions, for all tested MCS indices. On

\footnotetext{
${ }^{6}$ Given the steep transitions shown in PRR surfaces, PRR values between 0.3 and 0.7 are good indicators of the limits of link feasibility. Henceforth, for the purpose of these tests, we consider a link feasible for a given MCS if PRR > 0.5.
} 


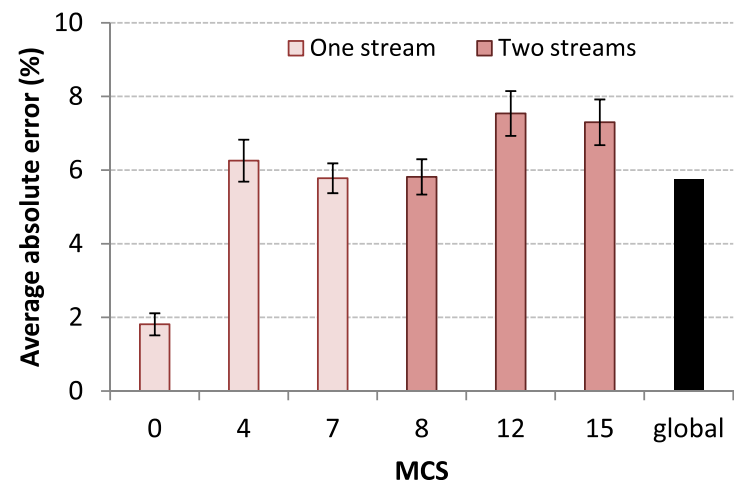

(a) Performance of link PRR predictions (99\% confidence intervals).

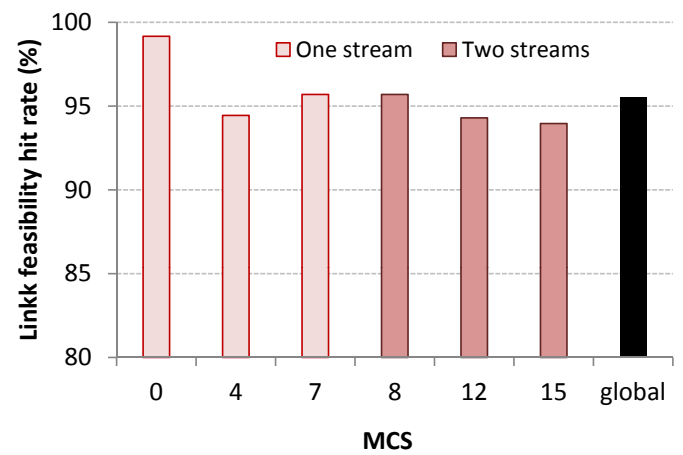

(b) Performance of link feasibility predictions.

Figure 11: Evaluation of the link predictor in new environments.

average, the error in our improved PRR predictions lies below 5.8\%. Fig. 11.(b) shows that link feasibility predictions improve to a $95.5 \%$ hit rate.

Recall that we interpolate to fill the gaps in a PRR(SNR, diffSNR) surface. We observe that regardless of whether the predictions come from interpolated or measured values, the predictor accuracy remains the same. For the measurements conducted in unfamiliar environments, we predict $71 \%$ of the indoor links from measured values while the remaining $29 \%$ come from interpolated values. For outdoor links, the proportion is 61 measured to $39 \%$ interpolated. For the familiar environment, interpolated values are not used. It is therefore not surprising that the outdoor environment has a greater number of predictions from interpolated values, as it exhibits different multipath characteristics from an indoor environment, where the PRR(SNR, diffSNR) 


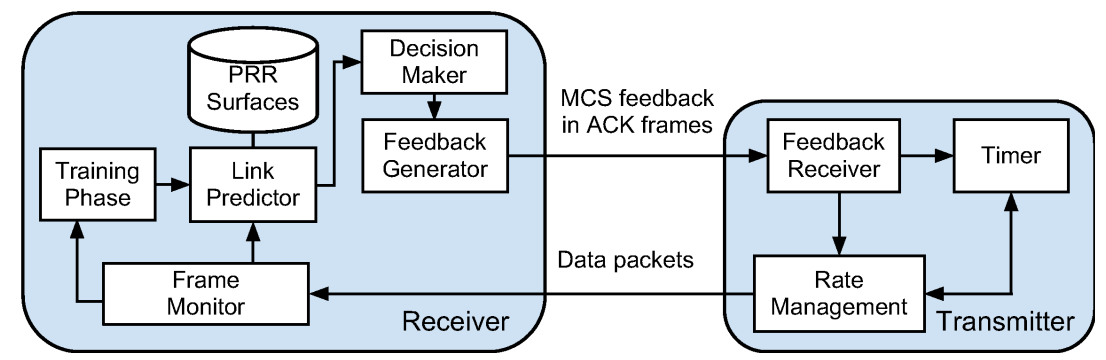

Figure 12: Block diagram of ARAMIS and the communication flow between its components implemented at the receiver (left block) and transmitter (right block).

360

surfaces were built.

A detailed evaluation of PRR predictions in these two new environments reveals the same patterns observed in the lab measurements. First, performance of aggressive modulations is more difficult to predict, and this is translated into lower feasibility prediction rates and higher PRR prediction errors. Second, PRR prediction errors increase when spatial multiplexing is used (4.6\% average absolute error for one stream vs $6.9 \%$ for two). Finally, the PRR of a $20 \mathrm{MHz}$ channel can be predicted with slightly greater accuracy than a $40 \mathrm{MHz}$ channel $(96.0 \%$ feasibility prediction hits for $20 \mathrm{MHz}$ channels vs $95.1 \%$ hits for $40 \mathrm{MHz}$ ).

Spatial multiplexing, aggressive modulations, and wider (i.e. $>20 \mathrm{MHz}$ ) channels are all features of new generation IEEE WLANs that achieve higher data rates at the risk of greater susceptibility to loss and changes in environment conditions. Accurately reflecting such detailed environment conditions however, such as the number of independent paths and frequency selectivity, is achieved using fine-grained CSI alone. Our link predictor, based on diffSNR forgoes the high cost of fine-grained CSI for ease of implementation, by using coarse-grained information on channel conditions, while maintaining a reasonable level of accuracy in predicting environment conditions. In the remainder of this paper, we prove the utility of diffSNR by implementing a novel close-loop rate adaptation mechanism that includes the link predictor described in this section. 


\subsection{Overview}

ARAMIS is a closed-loop RA solution for 802.11n MIMO environments. In the design of such a solution, we identify three important, high-level components. These three components form the critical foundation towards the implementation of ARAMIS. link metric, diffSNR. Current channel status information is used as input to the Link Predictor, which estimates the PRR of the link for all supported MCS and bandwidth combinations. As explained in Section 3 , our measurement-based Link Predictor needs 


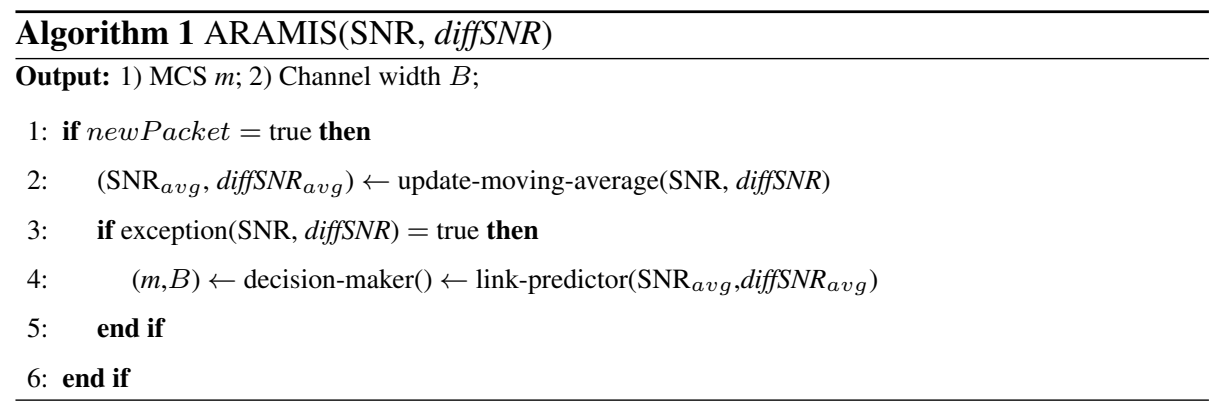

access to the set of pre-computed PRR surfaces. To improve the accuracy of predictions, the Link Predictor is assisted by a Training Phase that corrects errors in predicted PRR values in real-time by comparing recent predictions with current performance reported by the Frame Monitor. The Decision Maker then takes the PRR predictions from the Link Predictor, and based on some performance model, selects the best operating point. Using a standard-compliant mechanism, the Feedback Generator encapsulates information on the best possible rate in ACK frames to be sent to the transmitter. Finally, the transmitter's Feedback Receiver forwards the selected MCS and bandwidth to a Rate Management entity, which configures the PHY accordingly. In the absence of feedback, a backup Timer is implemented at the transmitter to reset the operating rate.

Following this outline, we now describe in detail the design components of our rate selector, which is used as a framework for the implementation of RA with diffSNR (including the diffSNR-based link predictor).

\subsection{Rate Selector}

The rate selector is the final main and all-inclusive design component of ARAMIS. An effective rate selector in a closed-loop, 802.11 RA model identifies changes in environment conditions and responds with the appropriate rate using a standard-compliant feedback method. To achieve these goals, we now describe how we combine our knowledge of our link metric, in this case (SNR, diffSNR), and the Link Predictor in the design of an effective rate selector. We use the terminology illustrated in Fig. 12 . 


\subsubsection{Frame Monitor}

The first step of a rate selector is to identify changes in channel conditions. This step is necessary to determine when an alternative rate might be appropriate. We have verified the accuracy of (SNR, diffSNR) in predicting link quality. We now describe how we monitor the behavior of per-packet (SNR, diffSNR) in real-time, using existing active traffic, to identify changes in channel conditions.

Fig. 5 depicts the evolution in per-packet (SNR, diffSNR) over time for a given link. Over a short period of time, (SNR, diffSNR) can fluctuate rapidly. To identify when changes in (SNR, diffSNR) could reflect a change in channel conditions, we apply an exponentially weighted moving average approach. ARAMIS stores (SNR, diffSNR) for every packet received and computes their moving average $\left(S N R_{\text {avg }}\right.$, diffSNR $\left.R_{\text {avg }}\right)$. We maintain moving averages not only for the average (SNR, diffSNR) values, but also for their standard deviation $\left(S N R_{s d}\right.$, diffSNR $\left.R_{s d}\right)$. ARAMIS initiates lookups to the link predictor if the current (SNR, diffSNR) lies outside of the range specified by $S N R_{a v g} \pm$ $S N R_{s d}$. The same conditions apply for diffSNR.

\subsubsection{Decision Maker}

Our rate selector uses a link's current channel conditions, reflected through the link metric, as input arguments to the Link Predictor, in this case using $\left(\mathrm{SNR}_{\text {avg }}\right.$, diff$\left.S N R_{\text {avg }}\right)$. The Link Predictor determines accurate PRR estimates for all supported MCS and bandwidths for that link, as described in Section 3 The role of the Decision Maker is to use this information to select the MCS and bandwidth configuration that yields the highest throughput. One model would be to select the configuration with the highest expected throughput. The computation of the expected throughput, however, requires a foreknowledge of the packet size implemented at the transmitter [10], which is not available at the receiver. Furthermore, this approach adds significant overhead to the computation of the appropriate rate. 


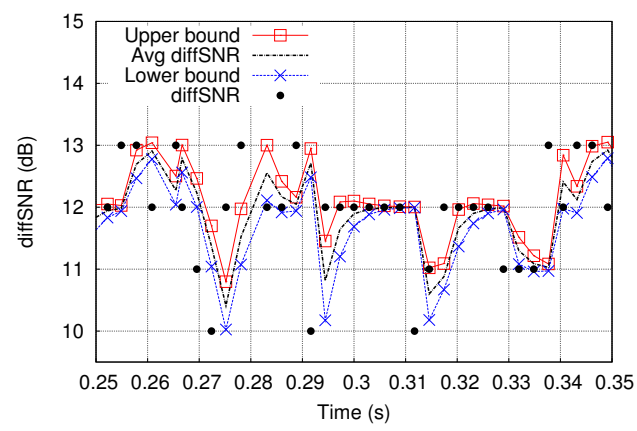

(a) Variation of diffSNR with time.

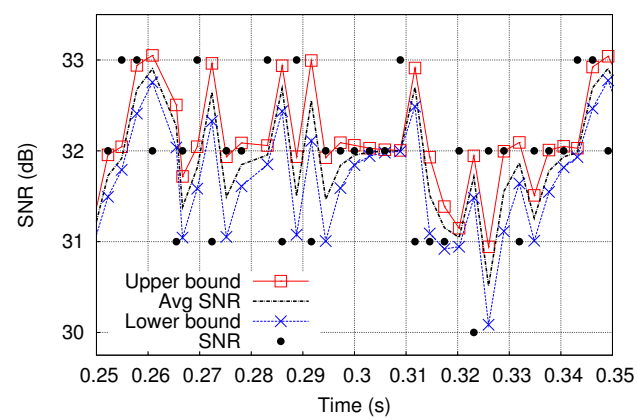

(b) Variation of SNR with time.

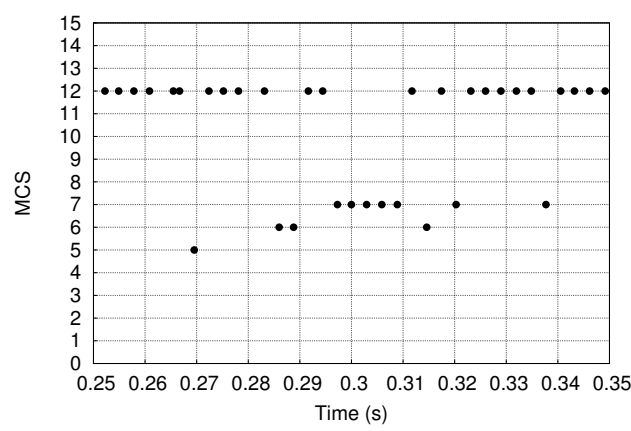

(c) Selected rate based on diffSNR, SNR values from previous packet measurements.

Figure 13: Depiction of ARAMIS measurements and behavior. We show that ARAMIS responds to changes in (SNR, diffSNR) conditions by modifying the MCS and bandwidth when necessary. In this case, the link always chooses a $40 \mathrm{MHz}$ channel.

We adopt a simple yet effective approach. Our model selects the MCS and bandwidth combination with the highest PHY bitrate from a reduced set of combinations whose predicted PRR is above a threshold. By adjusting this threshold, ARAMIS 


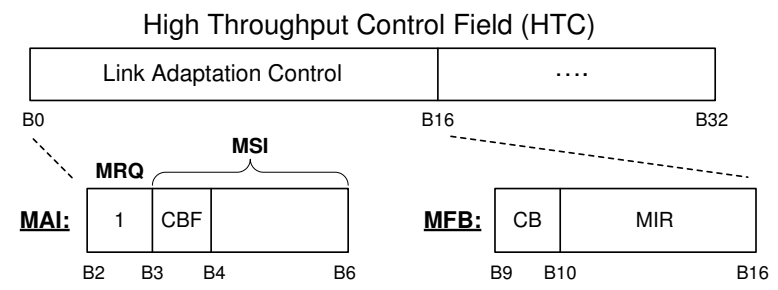

Figure 14: 802.11n compliant MCS feedback system.

Table 1: HTC subfields that support receiver-based RA.

\begin{tabular}{|c|c|}
\hline MRQ & MCS feedback request \\
\hline MSI & MRQ sequence identifier \\
\hline MFB & MCS feedback \\
\hline $\mathrm{CBF}^{*}$ & AP Channel bonding friendly \\
\hline $\mathrm{MIR}^{*}$ & Client MCS index request \\
\hline $\mathrm{CW}^{*}$ & Client channel width request \\
\hline
\end{tabular}

*: Bits allocated to support channel width feedback

has the flexibility to adapt to environments with varying error tolerances (increased to meet the requirements of reliability demanding applications, or relaxed to increase raw throughput).

Fig. 13 demonstrates the behavior of ARAMIS in real-time, as described in Algorithm 1. In Figs. 13 (a) and (b), we plot the instantaneous values, moving averages, and upper and lower bounds for our link metrics, both SNR and diffSNR. Fig. 13.c) depicts how ARAMIS changes MCS on a per-packet basis based on the correlated (SNR, diffSNR) values, where ARAMIS selects the MCS with the highest bitrate from those MCS that achieve a PRR above a given threshold for the current channel conditions. The corresponding bandwidth graph is not shown as ARAMIS always opts for a 40MHz channel for this link. Although not depicted, ARAMIS opted for a $20 \mathrm{MHz}$ channel, particularly for the weakest links in our evaluation. 


\subsubsection{Training Phase}

To improve the accuracy of predicted PRR values for all MCS and bandwidth combinations, a training mechanism is performed on-the-fly using the statistics of received frames, which does not incur any extra overhead. ARAMIS measures the link's actual PRR by dividing the number of received frames with the Retry flag set to 0 , by the total number of frames sent, the latter computed using frame sequence numbers. If aggregation is enabled, more precise PRR estimation could be provided by inspecting the bitmap field present in the Block ACK. ARAMIS then uses this measured PRR to update $E_{k}^{m, B}$ values in Eq. 1 In our implementation, $E_{k}^{m, B}$ is computed as the moving average of error samples with $\alpha=0.9$. Our $\alpha$ is large to give more weight to recent error samples, since the long-term mean error in PRR predictions is close to 0 .

\subsubsection{Feedback Generator}

We have discussed how ARAMIS identifies an appropriate rate given the current channel conditions. This rate, however, should be sent as feedback to the transmitter using a standard-compliant mechanism. To fully exploit variations in a MIMO channel, the 802.11n standard supports MCS feedback (MFB) in link adaptation [9]. MFB is a subfield of the HT Control field (HTC). HTC is a 4B optional field added to control packets (such as ACKs and Block ACKs).

Fig. 14 shows the HTC field with its corresponding link adaptation control field, where the subfields are described in Table 1 . We propose utilizing the unused fields and creating subfields that control bandwidth feedback. These added subfields allow ARAMIS to operate in conjunction with a channel management solution [11], where the CBF field set by the AP defines the supported bandwidth in the given WLAN. This allows ARAMIS to make informed channel width decisions using the insight from network layer conditions. For example, if $\mathrm{CBF}$ is set to 1 by a channel management approach, the client can request to operate on both a $20 \mathrm{MHz}$ and $40 \mathrm{MHz}$ channel, which it specifies in the $\mathrm{CW}$ subfield, and if $\mathrm{CBF}$ is set to 0 , the client only operates on a $20 \mathrm{MHz}$ channel. It is worth noting that the emerging 802.11ac standard supports such a client-based bandwidth adaptation mechanism, given the maximum supported bandwidth at the AP. 


\subsubsection{Timer}

A transmitter stops receiving feedback when the ARAMIS receiver does not receive transmitter frames. This can happen for two reasons. First, channel conditions at any given time could change drastically such that the PRR for the PHY configuration in use suddenly drops to 0 . Second, the transmitter may not have traffic to send. In both cases, the communication could be set at the wrong configuration with outdated information, since the transmitter is not receiving feedback to identify the appropriate MCS and bandwidth. This can lead to performance degradation. To mitigate this problem, we use a timer at the transmitter, whereby if feedback packets are not received before the timer expires, the MCS is set back to a reliable rate, MCS 8, then MCS 0 after a consecutive timeout, at the same bandwidth 77 Our results show that ARAMIS's perpacket rate adaptation is able to rapidly recover from this MCS reset.

\section{Performance Evaluation}

We evaluate ARAMIS, first using simulation based on packet traces from our experimental platform. We then implement ARAMIS on a real testbed and compare its performance to that of existing RA solutions under various network conditions. The goal of the trace-driven simulation is to evaluate the design choices for ARAMIS, since it gives us the flexibility to reproduce environment conditions while evaluating the performance of various design parameters. Furthermore, the post-processing of these traces allows us to simulate an optimal (and impractical) algorithm to use as a benchmark for our approach.

In our testbed implementation, we evaluate ARAMIS under various scenarios, including interference, mobility, and hidden nodes. Our goal is to demonstrate the efficacy of ARAMIS in accurately responding to channel conditions compared to other popular 802.11n RA solutions. We measure performance in terms of achieved throughput.

\footnotetext{
${ }^{7}$ This timer presents a tradeoff: a small timer may hastily fall back to low rates in the presence of severe collisions, and a large timer may prevent ARAMIS from rapidly adapting to degradations in signal quality.
} 


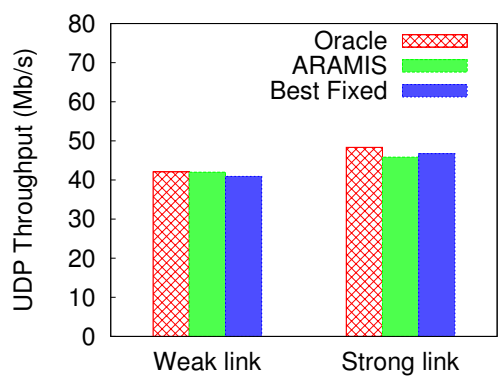

(a) Interference-free

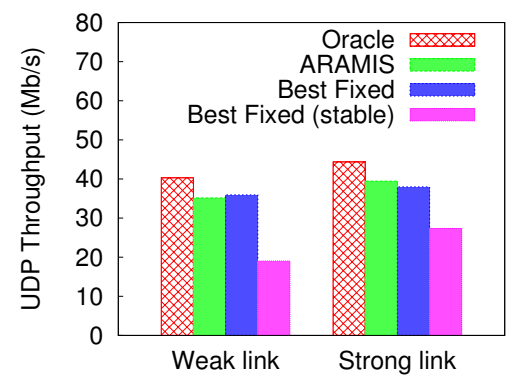

(b) Interference conditions

Figure 15: Comparison of ARAMIS against other representative solutions.

Testbed details: Both evaluation environments are built over our testbed platform that consists of 15 laptops deployed in both an open office and semi-open office environment. Each laptop is equipped with an 802.11n $2 \times 3$ MIMO PC card with an Atheros AR5416/AR5133 2.4/5GHz chipset. The AR5416 baseband and MAC processor allow MCS indices 0 to 15. Each laptop runs the Atheros Ath9k device driver that supports $802.11 \mathrm{n}[5]$. We run our experiments on the $5 \mathrm{GHz}$ range and verify the lack of background traffic with a spectrum analyzer.

\subsection{Trace-Driven Simulations}

\subsubsection{Simulation Environment}

The simulation utilizes packet traces that we collect over our testbed for various links. For each MCS, we send 5,000 1kB UDP datagrams from the AP to its client at a constant inter-packet delay of $2.7 \mathrm{~ms}$. We introduce this delay to avoid issues related to buffer overflow and conditions that restrict our ability to reproduce environment conditions. For the same reasons, we disable packet aggregation. The packet traces are stored at the client and consist of per-packet (SNR, diffSNR) values and inter-packet delays, as well as the computed PER, average SNR, and throughput for the entire transmission. We fix the transmit power to $11 \mathrm{dBm}$, which is the maximum common power level among all MCS. We conduct the above for both $20 \mathrm{MHz}$ and $40 \mathrm{MHz}$ channels.

We collect packet traces in interference-free environments as well as controlled in- 
terference conditions shown to affect $802.11 \mathrm{n}$ performance [10]. For the interference conditions, we introduce an interfering link that operates on either the same or an adjacent channel.

We use the above packet traces as input to our simulator. The simulator is built

in custom $\mathrm{C}$ and Python. The simulator works by replaying per-packet transmissions using each packet's transmission characteristics, namely its MCS, channel width, delay to the next consecutive packet, and packet loss.

We implement ARAMIS and other solutions, namely Best Fixed and Oracle in our simulator. Best Fixed fixes the MCS that maximizes throughput for the entire simulation run, and serves as a performance baseline. Best Fixed differs from ARAMIS in that it does not perform per-packet RA, but rather per-transmission RA by selecting the best MCS that maximizes throughput for that transmission. We add Best Fixed (stable) to the set of alternative solutions, and it represents the MCS Best Fixed chooses under stable, interference-free conditions. Finally, Oracle pre-processes the entire dataset of traces for every MCS and bandwidth combination and is thus able to select, for each packet, the fastest MCS that guarantees successful reception. Oracle makes optimal per-packet RA decisions, and therefore it serves as an upper bound for performance. Each simulation is run for 200 s of simulation time.

\subsubsection{Simulation Results}

Fig. 15 presents the simulation results. Fig. 15 a) depicts the UDP throughput under interference-free channel conditions for two types of links, where a weak link is unlikely to support high MCS due to weak SNR/diffSNR. Fig. 15.(b) shows the result with channel interference. We include Best Fixed (stable) to Fig. 15.b) to show how the best MCS that is selected in interference-free conditions would perform in interference environments. The insight from these results is the importance of per-packet rate adaptation in the presence of interference as well as in stable environments, where changes in the channel occur on narrow timescales. Best Fixed (stable) performs poorly when interference is introduced. ARAMIS takes advantage of per-packet processing, thus allowing quick and fine adjustments to varying channel conditions. In the presence of interference, however, there are fewer opportunities to take advantage of per-packet 


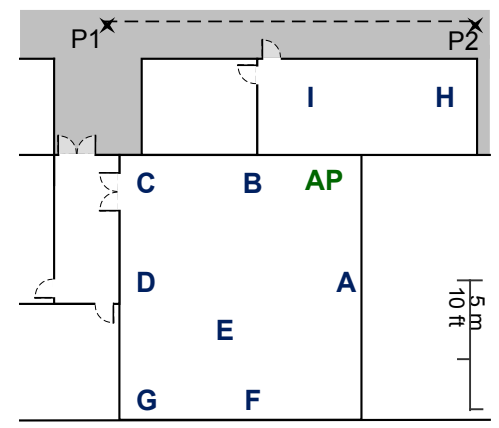

Figure 16: Floorplan of our testbed environment.

RA. As a result, ARAMIS is shown to provide near-optimal performance, similar to that obtained by the best fixed MCS, which is another ideal solution that requires foreknowledge of interference conditions to select the appropriate MCS. With aggregation disabled, all three solutions show little performance differences since they are close to the maximum theoretical throughput. In the next section we show how this PHY adaptation is combined with aggregation, a link layer feature, to leverage the potential of IEEE $802.11 \mathrm{n}$.

\subsection{Testbed Implementation}

\subsubsection{Testbed Environment}

We compare the performance of ARAMIS to that of two widely used open source 802.11n RA solutions, Ath9k [5] and Minstrel HT [4], and RAMAS [2], which was recently shown to be one of the best performing 802.11 n RA solutions.

We run RAMAS using the implementation made available by its authors. RAMAS is a credit-based system that divides the features of 802.11n RA into two groups: a modulation group, which consists of the 802.11n-supported modulation types, and an enhancement group that includes the number of independent spatial streams and bandwidth. RAMAS implements two independent credit-based systems for upgrading and downgrading the features of each group, where each group has a different set of rules for accumulating credits. For example, if the flow between a transmitter/receiver pair accumulates a number of credits within a given time window that exceeds a set thresh- 
old (where the credit counter is incremented by one each time packet errors fall below a given threshold), RAMAS switches to a more aggressive modulation (and vice versa).

Minstrel HT and Ath9k both use random sampling to find the best MCS. Minstrel HT, however, includes MCS with different bandwidths in its sampling group. Ath9k does not have a mechanism for enabling channel bonding, and to ensure a fair comparison, we set Ath9k's bandwidth to $40 \mathrm{MHz}$ to allow it to exploit higher data rates. Ath9k switches to a $20 \mathrm{MHz}$ channel when the PER is high. Other schemes select channel width based on their algorithm, and independently of the rate.

We evaluate the RA algorithms in a wide variety of scenarios, including interference and mobility. We fix transmit power to $11 \mathrm{dBm}$ and enable packet aggregation. We measure UDP throughput and PER, and average the results over 5 runs. The floorplan of our semi-open office, experimental environment is shown in Fig. 16, where the letters represent node locations. We note that this evaluation is based on a reduced set of 11 nodes. This reduced set is carefully chosen to include links with different characteristics (LoS, non-LoS, and a wide range of received signal strengths).

In our implementation of ARAMIS, we faced restrictions where the available chipset code does not support enabling an HTC field for $802.11 \mathrm{n}$ feedback. We mitigate this issue by implementing netlink sockets and transmitting packets with the HTC field over the wire from the receiver to the transmitter driver code. As a result, we believe the performance in our evaluation is a lower bound. Although transmitting feedback over the wired ensures no delivery loss, note that if a packet is not successfully received (e.g. there is a collision or loss), feedback will not be generated over the wire, as no ACK will be sent over the air. If the packet is successfully received, the loss of an ACK is unlikely as shorter ACK frames are sent at low, reliable rates, while the feedback over the wire is always received with a larger delay. The overhead of user-space-kernel communications, though minimal, often lead to delayed rate feedback receptions that trigger timeouts that mimic ACK packet losses.

Moreover, the devices do not provide open access to the hardware generated BlockACK at the receiver. This leads to inaccurate PER measurements, which reduces the precision of the ARAMIS training mechanism, explained in Section 4.2.3, and the accuracy of measured $E_{k}^{m, B}$ samples. 


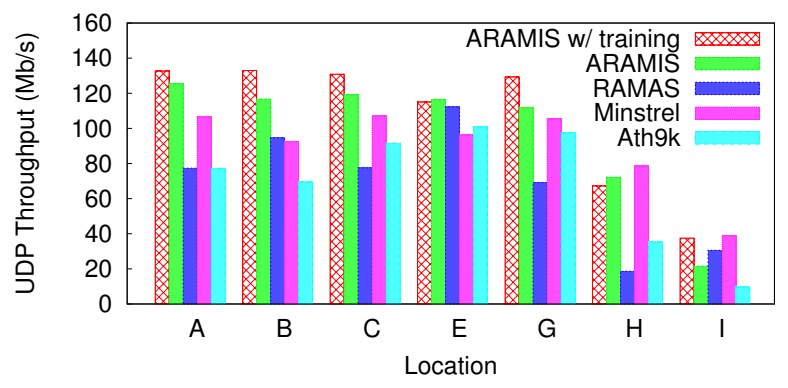

Figure 17: Algorithm performance in an interference-free environment.

\subsubsection{Testbed Results}

Figs. 17 and 18 show that ARAMIS consistently outperforms other algorithms in all test cases, with an average of 0.62 fold and up to a 2 fold throughput increase in interference-free environments, an average of 2 fold and up to a 10 fold increase in interference conditions, and a $25 \%$ increase in mobile environments.

Interference-free: To assess how well each algorithm handles random channel loss, for example due to shadowing or multipath, Fig. 17 shows the performance in an interference-free environment at seven different locations. Even without the training mechanism, ARAMIS outperforms other algorithms with throughput gains of up to $26 \%$ and an average of $16 \%$ over Minstrel HT, up to $124 \%$ and an average of $90 \%$ over Ath9k, and up to $287 \%$ and an average of $79 \%$ over RAMAS. Note that our results for RAMAS are somewhat different from those reported [2], since they were obtained in different scenarios. RAMAS was previously evaluated only on the $2.4 \mathrm{GHz}$ range, which significantly limits the performance benefits of $802.11 \mathrm{n}$ features [22, 3].

RAMAS leads to an average PER of $11 \%$ and a maximum of $20 \%$. The credit scheme it uses to adapt the number of streams is conservative, while the scheme to adapt the modulation and coding is aggressive. This mismatch causes RAMAS to often operate at sub-optimal rates with high modulations and single stream (e.g. MCS 7), which leads to high PER and reduced performance. Ath9k and Minstrel HT's random sampling incurs high overhead that results in poor performance. Ath9k also assumes PER monotonically increases with rate, which causes it to seek a very low PER region 


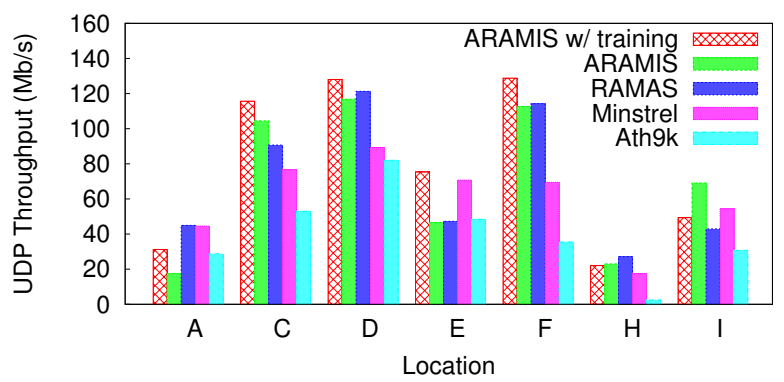

(a) Adjacent $40 \mathrm{MHz}$ interferer

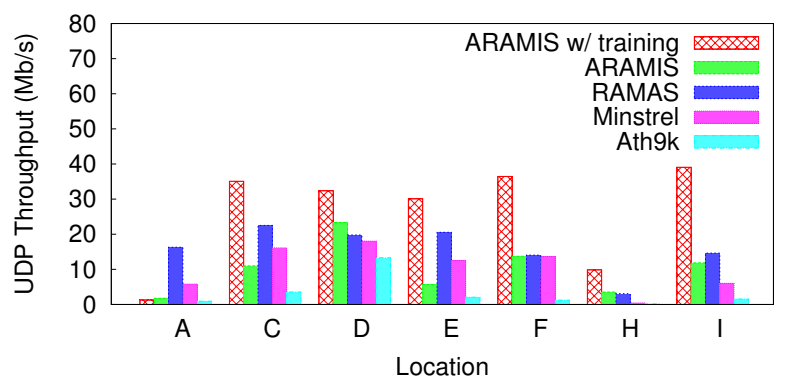

(b) Adjacent $20 \mathrm{MHz}$ interferer

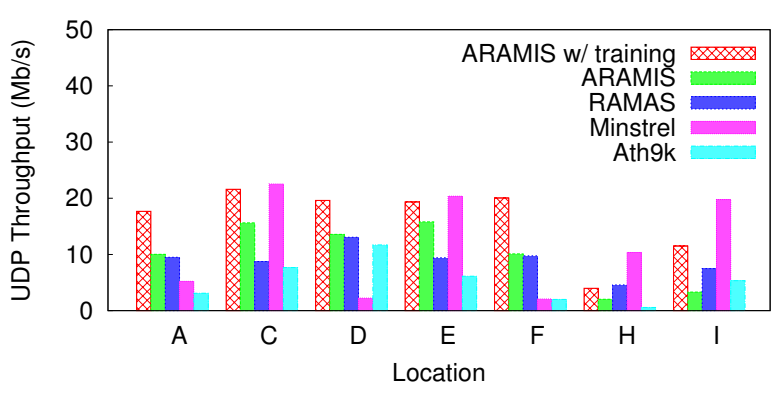

(c) Channel sharing with a $20 \mathrm{MHz}$ Interferer.

Figure 18: Algorithm performance under interference conditions. 
(between 2 and 5\%) at the cost of often ignoring suitable high rates.

ARAMIS relies on our link predictor for rate selection and hence does not require random sampling. Its link prediction accuracy and ability to adapt MCS and bandwidth on a per-packet basis maximize opportunities to exploit more aggressive rates without sacrificing PER. We observe an average PER between 4 and 6\%. ARAMIS is therefore suitable for low error tolerance applications, such as online gaming and bulk file transfers.

Interference: We now assess how the algorithms perform under interference from signal leakage, hidden nodes, and channel sharing.

Signal leakage is produced by transmissions on adjacent channels and can result in collisions similar to the hidden node problem. We evaluate how the algorithms react to interference due to leakage with varying interferer bandwidth, as we discovered that the impact of leakage varies according to channel width [10]. Fig. [18 (a) presents results with an interfering link that operates on an adjacent $40 \mathrm{MHz}$ channel, while Fig. 18.b) for an adjacent $20 \mathrm{MHz}$ interferer.

Ath9k and Minstrel HT respond frequently and rapidly to interference by reducing the rate. Reducing the rate exacerbates the impact of leakage; frame transmission time increases and so do the opportunities for collisions. Similarly, RAMAS responds to channel disturbances by first reducing the number of streams, thus reducing the transmission rate.

With signal leakage, the reported SNR may be low and collisions could be interpreted as wireless losses. ARAMIS's PRR predictions hence may not match the measured values from the training mechanism. When the prediction error $E_{k}^{m, B}$ exceeds a given threshold, which we set to 0.2 based on our experiments, ARAMIS interprets that there is a collision problem and limits the influence of the training mechanism; it sets $E_{k}^{m, B}$ to the maximum allowed value, thus maintains transmissions at suitable high rates. For an adjacent $40 \mathrm{MHz}$ interferer shown in Fig. 18 (a), we improve the throughput by an average of $10 \%$ and up to $60 \%$ over RAMAS, an average of $25 \%$ and up to $85 \%$ over Minstrel HT, and an average of $192 \%$ and up to $782 \%$ over Ath9k. For an adjacent $20 \mathrm{MHz}$ interferer shown in Fig. 18 b), the improvement is an average of 
$88 \%$ and up to $220 \%$ over RAMAS, an average of $400 \%$ and up to $412 \%$ over Minstrel HT, and an average of $1900 \%$ and up to $1908 \%$ over Ath9k. We observe greater performance improvements with an adjacent $20 \mathrm{MHz}$ interferer, since it is the more harmful configuration [10], and ARAMIS mitigates this interference.

Although the cap on the error threshold mitigates the effect of interference on RA, it can also degrade performance, as seen from location A in Fig. 18,b): ARAMIS first selects a rate which it identifies is best under interference-free conditions, and then reacts to high losses by capping the error threshold to 0.2 . We find that, in cases such as location A when a strong link suddenly becomes extremely lossy due to strong interference from channel leakage (or other non-802.11 interference), the error threshold forces ARAMIS to stay at higher rates than best, leading to performance losses.

We also investigate the channel sharing scenario with an interferer on a $20 \mathrm{MHz}$ channel. This scenario has been shown to create worse fairness issues than a $40 \mathrm{MHz}$ co-channel interferer whereby the slower $20 \mathrm{MHz}$ channel occupies the medium for longer periods of time [10]. In Fig. 18.c), we evaluate how well the algorithms perform under such conditions.

The presence of co-channel interference slightly increases collision probability, and thus $E_{k}^{m, B}$ increases, but remains under its maximum allowed value. As a result, the probability of using high rates is slightly reduced and Minstrel HT matches ARAMIS's performance in some locations since those collisions seldom affect Minstrel's random probing mechanism. At locations $\mathrm{H}$ and I in Fig. 18(c), we notice that channel sharing coupled with poor channel conditions can hamper the performance of ARAMIS. Channel sharing limits the number of opportunities to transmit, and if channel conditions are already poor and most transmissions are lost, this phenomenon can trigger ARAMIS's expiration timer, leading to frequent fall-backs to slow MCS. This phenomenon motivates the need for a dynamic expiration timer based on channel conditions.

The timely detection and adaptation to the channel conditions give ARAMIS an advantage over other algorithms, and this advantage is also evident in channel sharing conditions. At all locations, ARAMIS maintains the high order rates, thus exploiting its available channel time. ARAMIS improves the throughput by up to $76 \%$ over RAMAS, $251 \%$ over Minstrel HT, and $366 \%$ over Ath9k. 
Mobility: We create a mobility scenario to evaluate the responsiveness of ARAMIS

other $802.11 \mathrm{n}$ features, such as channel bonding [28, 26], or consider alternative energy efficiency goals [25]. 


\section{Conclusion and Future Work}

The 802.11n standard has been touted as a new revolution in Wi-Fi technology,

740

750

755

be applied in the context of the emerging 802.11ac standard, where MCS and channel width selection are faced with further challenges.

\section{Acknowledgments}

This work is partially supported by the National Science Foundation under Grant 765

o. 1032981. Any opinions, findings, and conclusions or recommendations expressed in this material are those of the author(s) and do not necessarily reflect the views of 
the National Science Foundation. This work is also partially supported by the Spanish Government through project TEC2012-32531 and Programa Nacional de Movilidad de Recursos Humanos del Plan Nacional de I-D+i 2008-2011.

\section{References}

[1] R. Crepaldi, J. Lee, R. Etkin, S.-J. Lee, R. H. Kravets, CSI-SF: Estimating wireless channel state using CSI sampling and fusion, in: IEEE Infocom, 2012.

[2] D. Nguyen, J. Garcia-Luna-Aceves, A practical approach to rate adaptation for multi-antenna systems, in: IEEE ICNP, 2011.

[3] I. Pefkianakis, Y. Hu, S. H. Wong, H. Yang, S. Lu, MIMO rate adaptation in 802.11n wireless networks, in: ACM MobiCom, 2010.

[4] Minstrel HT Linux Wireless, "http://linuxwireless.org/en/developers".

[5] M. Wong, J. M. Gilbert, C. H. Barratt, Wireless LAN using RSSI and BER parameters for transmission rate adaptation, US patent 7,369,510 (2008).

[6] L. Deek, E. Garcia-Villegas, E. Belding, S.-J. Lee, K. Almeroth, Joint rate and channel width adaptation for 802.11 MIMO wireless networks, in: IEEE Secon, 2013.

[7] D. Halperin, W. Hu, A. Sheth, D. Wetherall, Predictable 802.11 packet delivery from wireless channel measurements, in: ACM SigComm, 2010.

[8] S. H. Y. Wong, H. Yang, S. Lu, V. Bharghavan, Robust rate adaptation for 802.11 wireless networks, in: ACM MobiCom, 2006.

[9] IEEE 802.11n-2009 Amendment 5: Enhancements for Higher Throughput, IEEE-SA (Oct. 2009).

[10] L. Deek, E. Garcia-Villegas, E. Belding, S.-J. Lee, K. Almeroth, The impact of channel bonding on 802.11n network management, in: ACM CoNEXT, 2011. 
[11] L. Deek, E. Garcia-Villegas, E. Belding, S.-J. Lee, K. Almeroth, Intelligent channel bonding in 802.11n WLANs, IEEE Transactions on Mobile Computing 13 (6) (2013) $1242-1255$.

[12] C. Reis, R. Mahajan, M. Rodrig, D. Wetherall, J. Zahorjan, Measurement-based models of delivery and interference in static wireless networks, in: ACM SigComm, 2006.

[13] J. Camp, E. Knightly, Modulation rate adaptation in urban and vehicular environments: cross-layer implementation and experimental evaluation, in: ACM MobiCom, 2008.

[14] D. Aguayo, J. Bicket, S. Biswas, G. Judd, R. Morris, Link-level measurements from an 802.11b mesh network, in: ACM SigComm, 2004.

[15] A. Amini, D. Lu, C. Edelman, Effects of high peak-to-average ratio on $5 \mathrm{GHz}$ WLAN power amplifier, in: DesignCon, 2002.

[16] H. Yang, A road to future broadband wireless access: MIMO-OFDM-based air interface, IEEE Communications Magazine 43 (1) (2005) 53-60.

[17] M. Y. Arslan, K. Pelechrinis, I. Broustis, S. V. Krishnamurthy, S. Addepalli, K. Papagiannaki, Auto-configuration of 802.11n WLANs, in: ACM CoNext, 2010.

[18] E. Aryafar, N. Anand, T. Salonidis, E. W. Knightly, Design and experimental evaluation of multi-user beamforming in wireless LANs, in: ACM MobiCom, 2010.

[19] D.-S. Shiu, G. Foschini, M. Gans, J. Kahn, Fading correlation and its effect on the capacity of multielement antenna systems, IEEE Transactions on Communications 48 (3) (2000) 502-513.

815 [20] D. Tse, P. Viswanath, Fundamentals of Wireless Communication, Cambridge University Press, 2005. 
[21] M. Pursley, D. Taipale, Error probabilities for spread-spectrum packet radio with convolutional codes and viterbi decoding, IEEE Transactions on Communications 35 (1) (1987) 1-12.

[22] V. Shrivastava, S. Rayanchu, J. Yoonj, S. Banerjee, 802.11n under the microscope, in: ACM IMC, 2008.

[23] M. Vutukuru, H. Balakrishnan, K. Jamieson, Cross-layer wireless bit rate adaptation, in: ACM SigComm, 2009.

[24] W. H. Xi, A. Munro, M. Barton, Link adaptation algorithm for the IEEE 802.11n MIMO system, in: Networking LNCS, 2008.

[25] C.-Y. Li, C. Peng, S. Lu, X. Wang, Energy-based rate adaptation for 802.11n, in: ACM Mobicom, 2012.

[26] R. Combes, A. Proutiere, D. Yun, J. Ok, Y. Yi, Optimal rate sampling in 802.11 systems, in: IEEE Infocom, 2014.

[27] F. Peng, J. Zhang, W. E. Ryan, Adaptive modulation and coding for IEEE 802.11n, in: IEEE WCNC, 2007.

[28] W. Kim, O. Khan, K. Truong, S.-H. Choi, R. Grant, H. Wright, K. Mandke, R. Daniels, R. Heath, S. Nettles, An experimental evaluation of rate adaptation for multi-antenna systems, in: IEEE Infocom, 2009. 